\title{
MOORE SPACES AND UNIFORM SPACES
}

\author{
F. BURTON JONES ${ }^{1}$
}

Although arising from different motivations Moore spaces $^{2}$ and uniform spaces [7] are generalizations of metric spaces which have considerable similarity. This can be seen from the following characterizations by means of open coverings:

Let $S$ be a regular Hausdorff space.

If there exists a family $\{G\}$ of open coverings of $S$ such that

(a) $\{G\}$ is countable and ${ }^{3}$

(b) if $U$ is an open set and $p \in U$, there is an element $G$ of $\{G\}$ such that $G_{p}{ }^{*} \subset U$, then $S$ is a Moore space and conversely [6].

If there exists a family $\{G\}$ of open coverings of $S$ such that

$\left(\mathrm{a}^{\prime}\right)$ if $G_{1}$ and $G_{2}$ are elements of $\{G\}$ there is an element $G_{3}$ of $\{G\}$ which is a star refinement of both $G_{1}$ and $G_{2}$ and

(b) if $U$ is an open set and $p \in U$, there is an element $G$ of $\{G\}$ such that $G_{p}{ }^{*} \subset U$, then $S$ is a uniform space ${ }^{4}$ and conversely [5].

Of course if a $\{G\}$ exists so that all three conditions (a), $\left(\mathrm{a}^{\prime}\right)$ and (b) hold true simultaneously, $S$ is metric. ${ }^{5}$ Except for the obvious fact that the Hausdorff first countability axiom must hold true for Moore spaces while it need not do so for uniform spaces, it is by no means obvious that they differ otherwise.

L. F. McAuley has given an example of a normal semi-metric Hausdorff space $S$ which is not a Moore space [3]. Being normal, $S$ is both regular and uniform and being semi-metric, the first countability axiom holds true for $S$.

I give below an example of a locally connected, connected, complete Moore space ${ }^{6}$ containing a point $p$ at which it is not completely regular. Hence it is not uniform [ 2 or 5 ]. This space is obtained by piecing together along their boundaries adjacent terms of a simple sequence

Presented to the Society, January 28, 1958; received by the editors November 9 , 1957.

1 A National Science Foundation Senior Postdoctoral Fellow.

2 A Moore space is a space satisfying Axiom 0 and parts (1), (2), and (3) of Axiom 1 of [4]. A complete Moore space is one satisfying Axioms 0 and 1 of [4].

${ }^{3}$ If $G$ is a covering of $S$ and $p$ is a point of $S, G_{p}$ denotes the collection of all elements of $G$ which contain $p$ and $G_{p}{ }^{*}$ denotes the sum of the elements of $G_{p}$.

4 More precisely, $S$ has a uniform structure whose topology agrees with the topology of $S$.

5 However there exist spaces which are both Moore spaces and uniform spaces but which are not normal and hence not metric. The space $\Lambda$ given below is such a space.

- Such a space will be arc-wise and locally arc-wise connected. 
of non-normal Moore spaces which converges to $p$.

THE SPACE $\Lambda$. Let the points of $\Lambda$ be the points on or below the $x$-axis in a Cartesian plane. Regions (i.e., basis elements for the topology of $\Lambda$ ) are of two types:

(a) if $p$ is a point of $\Lambda$ not on the $x$-axis, let the interior of a circle centered on $p$ and missing the $x$-axis be a region, and

(b) if $p$ is a point of the $x$-axis, let the point set consisting of $p$ plus the interior of an isosceles triangle with vertex at $p$ and base parallel (and below) the $x$-axis be a region when the vertex angle equals its adjacent sides.

The reader may easily verify using regions of rational diameter in constructing $\{G\}$ that:

(1) $\Lambda$ is a complete Moore space,

(2) $\Lambda$ is connected and locally connected and

(3) in $\Lambda$ the $x$-axis is a discrete point set.

Lemma. The $x$-axis is the sum of two mutually exclusive uncountable sets $A$ and $B$ such that if $U$ is an open set in $\Lambda$ containing uncountably many points of one of them, then $\bar{U}$ in $\Lambda$ contains uncountably many points of the other.

Proof. Express the $x$-axis as the sum of two mutually exclusive sets $A$ and $B$ such that any uncountable closed (in the usual topology of the $x$-axis) subset of the $x$-axis intersects both $A$ and $B$. Let $U$ be an open set in $\Lambda$ which intersects one of these sets, say $A$, in an uncountable set. Now suppose that $\bar{U} \cdot B$ is vacuous or countable. Define a function $f$ from the $x$-axis to the non-negative real numbers as follows: For each point $x$ of the $x$-axis let $f(x)$ be the altitude of some region in $\Lambda$ which contains $x$ but no point of $\Lambda-U$ (if such a region exists); otherwise let $f(x)$ be zero. As a function of a real variable (the usual topology for the $x$-axis) $f$ is continuous at every point of $B-\bar{U} \cdot B$ and discontinuous at each point of $U \cdot A$. Since the set of all points of discontinuity of $f$ is the sum of countably many closed (relative to the $x$-axis) sets, at least one of these closed sets $C$ is uncountable and intersects $B$ in at most countably many points. Hence some uncountable closed subset of $C$ fails to intersect $B$. This is a contradiction.

Construction of the space $\Lambda_{\infty}$. Let $\Lambda_{1}, \Lambda_{2}, \Lambda_{3}, \cdots$ be a simple infinite sequence of disjoint $\Lambda$-spaces congruent to $\Lambda$. (For convenience the reader may think of these as lying in different planes of 3 -space parallel to the plane of $\Lambda$.) For each $n$, let $H_{n}$ be the point set in $\Lambda_{n}$ which corresponds (by congruence) to the (arbitrary) point set $H$ in $\Lambda$. 
Points of $\Lambda_{\infty}$ are of three types:

(1) if $y$ is a point of $A_{1}+\sum_{1}^{\infty}\left[\Lambda_{n}-\left(A_{n}+B_{n}\right)\right], y$ is a point of $\Lambda_{\infty}$;

(2) if $n$ is odd and $x$ is a point of $B$, then the pair $\left(x_{n}, x_{n+1}\right)$ is a point of $\Lambda_{\infty}$; and if $n$ is even and $x$ is a point of $A$, then the pair $\left(x_{n}, x_{n+1}\right)$ is a point of $\Lambda_{\infty}$;

(3) finally adjoin one "ideal" point $p$ (distinct from all the above) to $\Lambda_{\infty}$.

Regions (i.e., elements of a basis for the topology of $\Lambda_{\infty}$ ) are of three types:

(1) if $y$ is a point of type 1 belonging to $\Lambda_{n}$, a region in $\Lambda_{n}$ containing $y$ but not intersecting a point of type 2 is a region in $\Lambda_{\infty}$;

(2) if $\left(x_{n}, x_{n+1}\right)$ is a point of type 2 and $R_{n}$ and $R_{n+1}$ are regions in $\Lambda_{n}$ and $\Lambda_{n+1}$ containing $x_{n}$ and $x_{n+1}$ respectively, then the set consisting of $\left(x_{n}, x_{n+1}\right)$ together with the points of $\left(R_{n}-x_{n}\right)+\left(R_{n+1}-x_{n+1}\right)$ is a region in $\Lambda_{\infty}$; and finally

(3) for each $n, p$ together with the points of $\Lambda_{\infty}$ of types 1 and 2 which do not intersect $\sum_{i=1}^{n} \Lambda_{i}$ is a region $R_{n}$ of $\Lambda_{\infty}$.

Now one may verify that the space $\Lambda_{\infty}$ has the following properties:

(1) $\Lambda_{\infty}$ is a complete Moore space;

(2) $\Lambda_{\infty}$ is connected and locally connected;

(3) the boundaries $C_{n}$ of regions $R_{n}$ containing $p$ form a sequence $C_{1}, C_{2}, C_{3}, \cdots$ converging to $p$;

(4) $\Lambda_{\infty}$ is completely regular at every point except $p$; but

(5) $\Lambda_{\infty}$ is not completely regular at $p$.

Proof of (5). Suppose that there exists a continuous function $g$ from $\Lambda_{\infty}$ to the real number interval $[0,1]$ such that $g(p)=0$ and $g(y)=1$ for $y \in A_{1}$. Let $U_{1}=\{x: g(x)<1 / 2\}$. For some $n, C_{n}$ [see (3) immediately above] is a subset of $U_{1}$. It follows from the lemma that $\bar{U}_{1}$ contains uncountably many points of $C_{n-1}$. Let $U_{2}=\{x: g(x)$ $<3 / 4\}$; then $U_{2}$ contains uncountably many points of $C_{n-1}$ and $\bar{U}_{2}$ contains uncountably many points of $C_{n-2}$. Letting $U_{3}=\{x: g(x)$ $<7 / 8\}$ and continuing in this manner back toward $A_{1}$ (at most $n$ times) one sees that for uncountably many points $y$ of $A_{1}, g(y)<1$. This is a contradiction.

\section{REFERENCES}

1. F. Burton Jones, Concerning normal and completely normal spaces, Bull. Amer. Math. Soc. vol. 43 (1937) pp. 671-677.

2. John L. Kelley, General topology, New York, Van Nostrand, 1955.

3. Louis F. McAuley, A relation between perfect separability, completeness, and normality in semi-metric spaces, Pacific J. Math. vol. 6 (1956) pp. 315-326.

4. R. L. Moore, Foundations of point set theory, Amer. Math. Soc. Colloquium Publications, vol. 13, 1932. 
5. John W. Tukey, Convergence and uniformity in topology, Princeton, Princeton University Press, 1940.

6. C. W. Vickery, Axioms for Moore spaces and metric spaces, Bull. Amer. Math. Soc. vol. 46 (1940) pp. 560-564.

7. Andre Weil, Sur les espaces a structure uniform et sur la topologie générale, Actualités Scientifiques et Industrielles, no. 551, 1937.

The University of North Carolina and

The Institute for Advanced Study 